\title{
The Game of Reading: When Saccades and Fixations Play on a Seesaw
}

\author{
Carlo Aleci* \\ Department of Ophthamology, The Gradenigo Hospital, Turin, Italy \\ *Corresponding author: carlo.aleci@gradenigo.it \\ Received February 11, 2014; Revised February 27, 2014; Accepted February 28, 2014
}

\begin{abstract}
Although in experimental psychology the fixation/saccadic sequence is a main topic of debate, so far in the clinical ophthalmological field such an argument has raised relatively few interest. Nevertheless, studying the possible alterations of the saccadic rate and of the positioning of fixations when analyzing a visual scene may illuminate on the source of reading difficulty in a variety of patients, like dyslexic children or those affected by hemianopia as well as central visual field impairment. Recently, new light has been shed on the saccadic/fixation dynamics, both in the temporal and spatial domain. In addition, according to recent findings a visuoperceptive distortion could potentially affect the saccades/fixation sequence, making the matter much more complex than expected. The aim of this article is therefore to describe the saccadic/fixation seesaw under the temporal and spatial perspective and to disclose the possible influence of visuoperceptive impairments.
\end{abstract}

Keywords: saccades, fixations, magnocellular system, parvocellular system, spatial relationship perception, anisotropy

Cite This Article: Carlo Aleci, "The Game of Reading: When Saccades and Fixations Play on a Seesaw." American Journal of Medical and Biological Research, vol. 2, no. 2 (2014): 31-36. doi: 10.12691/ajmbr-2-2-1.

\section{Introduction}

The analysis of an image, that is the recognition of its different features, is made possible thanks to two fundamental phases: fixations and saccades. If the correct functioning of fixations and saccades is important in the perception of natural scenes, it is even crucial during the lexical task.

\section{Fixations and Saccades}

The essential requirement for correctly recognizing a syllable is its projection onto the central portion of the retina, the fovea. The fovea, in fact, is the sole region whose anatomical and functional characteristics are suitable for this task. This is the reason why the fovea is called the site of the distinct vision, but it is not enough: an additional requirement is that the stimulus remains projected on the fovea as long as to be fully and correctly processed. This interval is called fixation. Fixations, therefore, allow the syllable to stay still on the fovea for the least time required to be processed. Their average duration is $180 \mathrm{msec}$, but it can be far lower (till to 30-40 msec) o higher, approaching $450 \mathrm{msec}$ [1].

Since reading a sentence implies processing letters/syllables/words in fast sequence, the fixation should move methodically along the string.

Such a shift consists of rapid movements, called saccades (or FEM, Fast Eye Movements). Along the sentence, therefore, saccades divert the fixation of a syllable so as to redirect it toward the next one: thereby, the position of each fixation depends on the point the saccade stops at the end of its movement. This region is called the landing site (LS). Normally, the landing site should match the convenient viewing position (CVP) or preferred viewing position [2,3], that is to say the position that minimizes the duration of the incoming fixation. The CVP is function of the length of the word.

Finally, the term perceptive center of gravity (PCG) or optimum viewing position [4], previosly theorized as optic center of gravity by Findlay in 1982 [5] and by Ottes in 1984 [6], and then reconsidered by Crawford e Higham in dyslexics [7], refers to the ideal position for the word to be optimally processed. Generally it localizes about to its midpoint. The optimal situation would be exact correspondence between convenient viewing position and perceptive center of gravity, so that:

$$
\mathrm{LS}=\mathrm{CVP}=\mathrm{PCG}
$$

As a matter of fact, a discrepancy occurs between CVP and PGC.

To better understand the contribute of saccades and fixations in the lexical task, it is necessary to examine in depth the way saccades and fixations interact when reading.

\subsection{Properties of the Saccades}

The properties of the saccades are speed, direction, precision (i.e. the degree of correspondence between landing site and convenient viewing position), and latency. 
FEM's speed, that can reach $500^{\circ} / \mathrm{sec}$, increases till to halfway of the saccadic movement, then it gradually slows down till to "land" on the landing site [8]: the speed $\mathrm{X}$ space function can therefore be defined as non-monotonic.

FEMs direction mostly follows the order of the writing: in western languages saccades are mainly directed toward the right, that is forward, and are therefore called forward saccades. The average width of forward saccades is about $2^{\circ}$.

In a small percentage of cases FEMs move in the opposite direction: these FEMs are called regression or backward saccades. Their role is to take back the fixation on a previous syllable whenever its visual or cognitive processing turns out to be not adequate enough. Compared to forward saccades, regression saccades are shorter, being just about $1^{\circ}$ wide.

A second factor responsible for the triggering of backward saccades is the malpositioning of the landing site: if an imprecise forward FEM causes excessive discrepancy between its actual landing site and the convenient viewing position, a regression saccade aimed at correcting the positional error is likely to take place $[9,10]$. In this case, the greater is the difference between landing site and convenient viewing position, the higher is the probability that a backward saccade takes place and the shorter is its latency.

This probability is at its maximum if the convenient viewing position is at the beginning or (to a less extent) at the end of the word, whereas it is at its minimum if it is localized about the midpoint [11,12].

Even if abnormal positional coding related to imprecise FEM pattern is the main factor responsible of backward saccades, linguistic and cognitive factors would play a role as well $[8,16]$, since the proportion of backward saccades (generally lower than $15 \%$ [1,13]) increases in cases of demanding reading $[1,14,15]$.

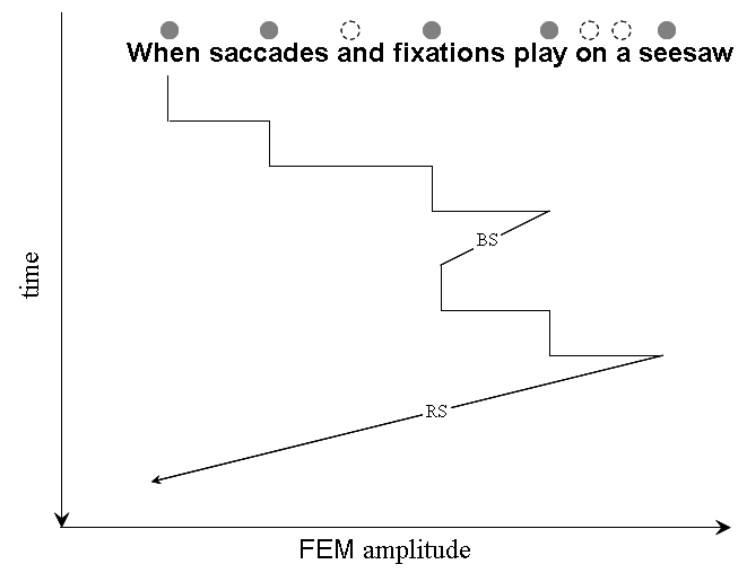

Figure 1. Schematic representation of the ocular movements when reading. In abscissa is FEM amplitude, in ordinate the fixation duration. The trend of the line represents the fixations/saccades alternation. The black circles refer to the landing sites, that in normal readers generally match the preferred viewing position. The dotted circles represent the skipped landing sites, since short words or high frequency words are generally processed parafoveally, therefore do not require fixation. Toward the end of the line a backward saccade (BS) takes place. At the end of the line a wide backward saccade (return sweep, RS) sets the fixation at the beginning of the next line

Remarkably, not every word needs to be fixated: short words like articles and prepositions and very common words (called high frequency words) are generally skipped by a proficient reader, and (pre)processed by the retinal region on the right side of the fovea (the parafovea).

What with forward saccades and backward saccades, fixation at least comes to the end of the line and it is repositioned at the beginning of the next line by a wide regression saccade, called return sweep (Figure 1).

\subsection{What Drives the Rhythm of the Fixation/Saccade Sequence?}

Since the early eighties, a number of studies has focused on the mechanisms managing the rhythm of the fixation/saccade sequence.

The three main speculative orientations are:

-the ones based on cognitive therories

-the ones based on oculomotor strategies

- the so-called theory of the pull-and-push.

According to the cognitive theories, fixation would stay still on the word for the bare minimum time needed for its full comprehension, then it is driven by the saccadic movement toward the next preferred viewing position [17].

According to the theory of the oculomotor strategy, the saccadic rhythm depends on a stereotyped model that becomes increasingly refined with the lexical experience. So, the stereotyped saccadic pattern is improved via corrective movements that the practice of reading makes more and more precise. The theory of strategy and tactics, in particular, assumes that the saccadic strategy aims at positioning the fixation close to the preferred viewing position on the word. Should the outcome not be satisfying, a refixation (corrective) movement (the tactic) adjusts the shot. With the lexical experience the reading strategy would get more and more precise, making the tactic less and less necessary [18].

Within this framework, the saccadic triggering can be considered as a stochastic event and the features of a word (i.e. its complexity) would affect the parameters controlling the probabilistic distribution of FEMs activation [19]. This way, reading experience would increase the likelihood of a FEM triggering after a $t$ interval for a given type of word and after a $t 1$ interval for a different type of word (e.g. a more complex one: in this case $t 1>t$ ), thereby optimizing the rhythm of the saccadic sequence as a function of the difficulty of the text.

\subsubsection{The Competition-Interaction Model}

In order to provide this hypothesis with a physiological basis, the pull-and-push model and its enhanced version, the competition-interaction model, have been advanced by Findlay \& Walker [20] and by Yang et al [21], respectively. According to these hypothesis, the rhythm of the saccade-fixation sequence is dictated by the competition between two different brain centers. More specifically, fixation maintenance and the subsequent saccadic activation depend on the interaction between central-vision-related fixate center and peripheral-visionrelated move center: when activated, the central-visionrelated fixate center, mediated by the parvocellular system, promotes fixation and helps its stability; in turn, the function of the peripheral-vision-related move center, mediated by the magnocellular system, is to promote the saccadic triggering. Being interconnected, when one center is activated, the other is proportionally inhibited. 
Based on this paradigm, an extrafoveal stimulus $S p$ activates the peripheral-vision-related move center that in turn inhibits progressively the central-vision-related fixate center in its concomitant processing a foveal stimulus $S f$. When, for the inhibitory effect of the former, the activation level of the latter drops below a threshold value, the fixation is suppressed, diverted by $S f$, and a saccade directed toward $S p$ takes place.

During the saccadic "jump", the process reverses: the activation of the central-vision-related fixate center rises progressively, inhibiting more and more the peripheralvision-related move center. When the activation level of the latter drops below the threshold, the jump comes to an end and the landing of the saccade takes place, allowing the fixation to consolidate on the next syllable ( $S p$ in this case).

The gradual rise of the activation of the fixate-center occurring about the end of the saccadic activation interval allows the onset of a refractory period, useful to avoid a sudden triggering of the next FEM: such a refractory period, therefore, prevents from untimely saccadic activation.

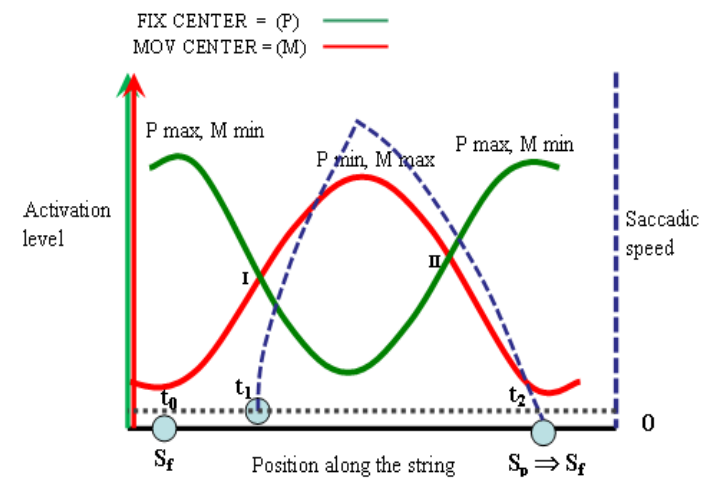

Figure 2. The saccade/fixation sequence according to the competitioninteraction theory. In abscissa (space scale) the foveal and parafoveal stimulus position $\left(\mathrm{S}_{\mathrm{f}}\right.$ and $\mathrm{S}_{\mathrm{p}}$, respectively) at time $\mathrm{t}_{0}$ (continuous axis) and time intervals $t_{0}, t_{1}, t_{2}$ (dotted axis) are depicted. In ordinate on the left is represented the activation level of the P-system corresponding to the central-vision-related fixate center (green) and of the M-system corresponding to the peripheral-vision-related move center (red). The ordinate on the left refers to the saccadic speed. The green and red curves describe the reciprocal interaction of the progressive activation of the two programming stations (green= fixation system=P-system; red= saccadic system=M-system). The blue dashed curve, referred to the blue dotted $\mathrm{x}$-axis (time scale), represents the saccadic speed. Note that the starting point of the saccade is the last fixation $S_{f}$. When the syllable $S_{\mathrm{f}}$ is fixated at time $t_{0}, P$ activation is at its maximum while $\mathrm{M}$ activation is at its minimum, resulting in a stable fixation. However, due to the presence of a second, peripheral syllable $\mathrm{S}_{\mathrm{p}}$, $\mathrm{M}$ activation rises more and more, gradually inhibiting the P-system until it matches and then overcomes its activation level (I) at time $t_{1}$. So, at time $t_{1}$ the prevailing effect of the peripheral-vision-related move center disrupts the fixation and promotes the saccadic triggering. FEM speed increases, keeping abreast of the Mactivation level up to a maximum, while the $\mathrm{P}$ system is concurrently inhibited. From that moment on, a new progressive P-activation of the central vision-fixate center starts to oppose the effect of the magnocellular peripheral move-center: it follows that the speed of the actual FEM lowers gradually, keeping abreast of the decreased magnocellular activation. When the activation level of the $\mathrm{P}$ system overcomes the $\mathrm{M}$ level (II), the speed of the saccade slows down further and its "landing" is forthcoming. The landing site in $S_{p}$ has become, therefore, the new fixation point (being now placed on the fovea). In the meanwhile, the complete $\mathrm{P}$ activation has overwhelmed the M-system, determining a total inhibition of the magnocellular peripheral movecenter. A new cycle takes place as soon as the $\mathrm{M}$ activation starts again to rise and at the same time $\mathrm{P}$ activation begins to decrease proportionally. The inhibition/interaction mechanism is open to cognitive modulation
Cognitive factors would have a modulatory function by increasing FEMs latency so as to prolong the fixation time: such an influence would account for increased reading time when coping with particularly demanding texts. It is noteworthy that a similar effect on the saccadic latency can result even from visuoperceptive difficulties [21].

Figure 2 shows the reciprocal activation/inhibition of the saccade/fixation system.

Two aspects are worth to be deepened: the timing of the saccadic triggering and the localization of the landing site: impaired triggering as well as imprecise localization of the landing site, in fact, may affect the lexical fluency.

\subsection{Timing of the Saccadic Triggering}

The time immediately before the activation of the saccade is critical (Figure 3): in fact, the latency of the FEM depends on what occurs in this interval. It has been reported that just after the landing of the FEM, therefore at the beginning of the fixation time, the magnocellular activation level is minimal since it is inhibited by the fixation (parvocellular) center. However, being a dynamic process, the M-inhibition fades gradually and at the same time the saccadic activation grows. After about $125 \mathrm{msec}$, the activation gradient gets steeper, till to reach a plateau. When the plateau is reached, the programming of the next FEM is completed and the imminent time of the saccadic triggering is a stochastic interval depending on the very moment the activation state fluctuates beyond the threshold [21]. The position of the plateau differs not only among the population but even within the same reader, depending on the level of difficulty of the text [21]: on the one hand, the inter-individual variability of the saccadic latency accounts for reading speed differences in the population. On the other hand, its intra-individual variability aims at optimizing the saccadic sequence as a function of the difficulty of the lexical material. So, for simpler texts the plateau localizes at higher level, making more likely the fluctuation of the activation level beyond the threshold (thereby facilitating FEMs activation), whereas for more demanding texts it sets lower, allowing longer fixations.

Saccadic latency generally lasts about 150-175 msec $[22,23,24,25]$ but it can vary greatly, so that FEMs can be classified into three types: early saccades, normal saccades and late saccades) [21].

Late saccades occur when the perception or the comprehension of the fixated word is unclear: in this case, the programmed forthcoming normal saccade is deleted and re-planned as late saccade. Since the activation of the late saccades is believed to depend also on the comprehension of the text, late saccades would be the only FEMs subject to cognitive modulation. Indeed, the effect the cognitive factor has on saccadic timing is supported by the finding that when an observer is asked to perform a cognitive task, namely to plan a sequence of saccades, the latency of the first movement is increased [26,27,28]. In this regard, it is noteworthy that increased latency of a saccade leads to increase of its accuracy [29,30].

On the other side, some early saccades show a very short latency (down to $80 \mathrm{msec}$ ). These particular FEMs, originally described in dyslexic readers and after recorded (even if to a less extent) also in normal readers [31], are called express saccades. Express saccades take place 
when the fixation centered on a target disrupts before the next target is presented [32]. For this reason, the occurrence of express saccades has been related to malfunctioning of the neurons in charge of the fixation (fixation neurons) [31,33] localized in the frontal eye fields [34,35], and to defective visual attention [36].
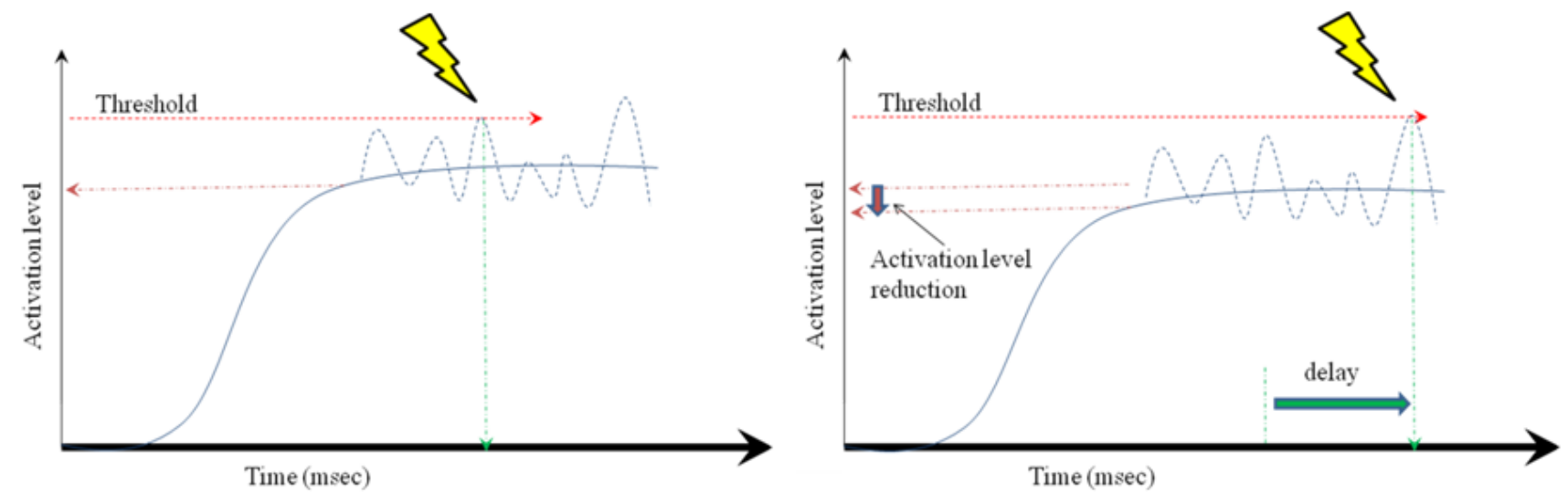

Figure 3. The saccadic activation level. At the beginning of the fixation time, the saccadic activation is at its minimum but tends to increase gradually. After about $125 \mathrm{msec}$ the trend gets faster raising up to a plateau. By now, the saccadic programming is completed and the saccadic triggering takes place as soon as the activation state fluctuates beyond the threshold. The placement of the plateau differs among individuals, explaining the reading differences in the population, as well as within the same reader, depending on the difficulty of the lexical material. For simpler texts the plateau would be higher (upper panel), helping the stochastic event leading to the saccadic triggering; on the contrary, for more demanding reading the plateau would be lower (bottom panel), hampering the saccadic activation, thereby allowing longer fixations

\subsection{Localization of the Landing Site.}

In addition to the timing of the saccadic triggering, a second crucial point deals with the placement of the landing site.

The frequency distribution of the landing site on a word is described by a gaussian function whose peak is the average position and the two tails refer to the standard deviation on the left (undershooting saccades) and on the right (overshooting saccades) [37].

The peak of frequency distribution of the landing site on the word tends to localize on the right of the perceptive center of gravity, but its position can vary considerably. The variability is mainly related to the oculomotor strategy [12], in particular to the tendency of the saccade to target the longer words, skipping the shortest ones. Evidently, in order for the FEM to select the words to target and the words to skip, the visual system needs to take a predefined length threshold as a reference. In this respect, the length estimate of the word would not depend on the number of characters it is made of, but on its spatial extent $[3,38]$. The boundary of the spatial extent of the word, in turn, is codified by the comparison between the spacing separating adjoining words and the spacing separating the letters of the words. To be "interword", such spacing has to be wider than the distance between the characters within each word.

Saccadic skipping concerns not only short words but also the commonest words ("high frequency words"), inferable from the reading context. So, like short words, also high frequency words do not need to be fixated (i.e. do not require to be targeted by a saccade) but can be processed parafoveally while the (foveal) fixation is engaged on the previous word. Evidently, this means that linguistic factors modulate the saccadic pattern as well $[39,40]$.

Still, word skipping is believed to be related even to visuoattentional mechanisms: the attentive shift on the next word $\left(\mathrm{P}_{1}\right)$ normally promotes a saccade $\mathrm{Sp}_{1}$ toward the word. If $\mathrm{P}_{1}$ is a short or high frequency word, the attentive shift would allow its parafoveal processing. In this case, three different possibilities referred to $\mathrm{Sp}_{1}$ can be hypothesized:

a) $\mathrm{Sp}_{1}$ is suppressed and after the parafoveal processing of $\mathrm{P}_{1}$ is completed, the visuoattentive focus shifts onto $\mathrm{P}_{2}$, promoting a new saccade directed toward $\mathrm{P}_{2}\left(\mathrm{Sp}_{2}\right)$.

b) $\mathrm{Sp}_{1}$ is triggered because its programming had been previously completed: in this case, $\mathrm{Sp}_{2}$ activates immediately after Sp1.

c) $\mathrm{Sp}_{1}$ is triggered but it is "restrained", "braked", so that it manages to overcome $\mathrm{P}_{1}$ but, does not reach $\mathrm{P}_{2}$ and localizes midway between the two words.

\section{Can Visual Perception Affect the Saccadic Pattern?}

In these oculomotor dynamics, the modulatory effect of the spatial extent of the words on the saccadic pattern deserves consideration. It could be, indeed, a factor of potential importance in the rehabilitation program of patients affected by visuoperceptive disabilities. The visuoperceptive element would have an effect on the oculomotor programming when reading, as abnormal spatial relationship perception of the text, leading to wrong estimate of the length of the words, could destabilize the saccadic/fixation alternance. It should be considered, indeed, that dyslexic subjects suffer from abnormal saccade/fixation sequence. Yet, such impairment is not found in extralexical tasks [7,41-50]. It could be argued, therefore, that in these patients defective saccade/fixation pattern rather than causative could more likely depend on abnormal coding of the word extent. According to this hypothesis, abnormal perception of the visual space filled by the sentence would bias the expected position of the perceptual center of gravity on the words, misguiding the landing sites of the saccades and consequent fixations. Indeed, in a previous work we have shown in a subgroup of dyslexic children abnormal spatial 
relationship perception with subjective contraction of the visual space along the horizontal and enlargement along the vertical coordinate [51]. We have defined this condition as vertical anisotropy [51,52], suggesting it could account for abnormal eye movements of dyslexics when reading.

This theoretical construct can be extended to other clinical areas, having a potential rehabilitative meaning for a number of ophthalmological diseases. For example, increased macular thickness in cases of age-related macular degeneration could lead to spatial relationship alteration on and around the fixation point, causing abnormal positional coding, thereby disruption of the normal saccadic pattern during the lexical task [53].

Apart from complex sensorimotor interactions, these considerations highlight the strict link between sensorial and oculomotor aspects of visual perception.

\section{Conclusion}

A main rule in the game of reading is the synchronization of the saccade/fixation seesaw: it is so delicate a mechanism that even a slight fault in its programming or execution may make reading fluently particularly demanding. Even in presence of adequate visual acuity, the perfectly balanced alternance of magnocellular and parvocellular activation might be disrupted or hampered by abnormal spatial relationship perception. A thorough analysis of these topics should therefore be provided in further investigations.

\section{Statement of Competing Interests}

The author has no competing interests.

\section{References}

[1] McConkie, G.W and Zola, D, Some characteristics in readers' eyes movements, in: Brain and Reading, von Euler, Lundberg \& Lennerstrand Editors, Macmillian Press Publishers, London, 1989.

[2] O’Regan, J.K, The convenient viewing hypothesis, in: Eye movements: cognition and visual perception. Monty, Senders \& Senders Editors, Erlbaum Publishers, Hillsdale, 1981.

[3] Rayner, K, "Eye guidance in reading: fixation localization within words," Perception, 8(1). 21-30. 1979.

[4] O’Regan, J.K., Jacobs, A.M, "Optimal viewing position effect in word recognition: a challenge to current theory,” J Exp Psychol Hum Percept Perform, 18(1). 185-197. 1992.

[5] Findlay, J.M, "Global vision processing for saccadic eye movements," Vis Res, 22(8). 1033-1045. 1982.

[6] Ottes, F.P, Van Gisbergen, J.A.M., Eggermont, J.J, "Metrics of saccades responses to visual double stimuli: two different modes," Vis Res, 24(10). 1169-1179. 1984.

[7] Crawford, T.J., Higham, S, "Dyslexia and the centre of-gravity effect,” Exp Brain Res, 137(1): 122-126. 2001.

[8] Rayner, K, "Eye movements in reading and information processing: 20 years of research,” Psychol Bull, 124(3). 372-422. 1998.

[9] Vitu, F., McConkie, GW., Zola D, About regressive saccades in reading and their relation to word identification, in: Eye Guidance in reading and scene perception, Underwood Editor, Elsevier Publishers, Oxford, 1998.

[10] McConkie, G.W., Kerr, P.W., Reddix, M.D., Zola, D., Jacobs, AM, "Eye movement control during reading: II. Frequency of refixating a word,” Percept Psychophys, 46(3). 245-253. 1989.
[11] Rayner, K., Sereno, S.C., Raney, G.E, "Eye movement control in reading: A comparison of two types of models," J Exp Psychol Hum Percept Perform, 22(5). 1188-1200. 1996.

[12] Reilly, R., O’Regan, JK, "Eye movement control in reading: a simulation of some word-targeting strategies," Vis Res, 38(2). 303-317. 1998.

[13] Rayner, K, "Eye movements in reading and information processing,” Psychol Bull, 85(3). 618-660. 1978.

[14] Jones, A., Stark, L, Abnormal Patterns of normal eye movements in specific dyslexia, in: Eye Movements in Reading: Perceptual and Language Processes. Rayner Editor, Academic Press Publishers, New York, 1983.

[15] Rayner, K., Pollatsek, A, Eye movements in reading: A tutorial review, in: Attention and performance XII: The psychology of reading, Coltheart Editor, Erlbaum Publishers, London, 1987.

[16] Hyönä, J., Pollatsek, A, "Reading Finnish compound words: Eye fixations are affected by component morphemes," J Exp Psychol Hum Percept Perform, 24(6). 1612-1627. 1998.

[17] Just, M.A., Carpenter, P.A, "A theory of reading: From eye fixations to comprehension,” Psychol Rev, 87(4). 329-354. 1980.

[18] O’Regan, J.K., Lévy-Schoen, A, Eye movement strategy and tactics in word recognition and reading, in: Attention and Performance XII: The psychology of reading, Coltheart \& Lawrence Editors, Erlbaum Publishers, Hillsdale, 1987.

[19] McConkie, GW., Dyre, BP, Eye fixation durations in reading: Models of frequency distributions, in: Reading as a perceptual process, Heller \& Pynte Editors, Elsevier Publishers, Oxford, 2000

[20] Findlay, J.M., Walker, R, "A model of saccade generation based on parallel processing and competitive inhibition,” Behav Brain Sci, 22(4). 661-674. 1999

[21] Yang, S.N., McConkie, G.W, "Eye movements during reading: a theory of saccade initiation times," Vision Research, 41(25-26). 3567-3585. 2001.

[22] Salthouse, T.A., Ellis, C, "Determinants of eye-fixation duration," Am J Psychol, 93(2). 207-234. 1980.

[23] Salthouse T.A., Ellis, C.L., Diener, D.C., Somberg, B.L, "Stimulus processing during eye fixations," J Exp Psychol Hum Percept Perform, 7(3). 611- 623. 1981.

[24] Rayner, K., Skowiaczek, M.L., Clifton, C., Bertera, J.H, "Latency of sequential eye movements: Implications for reading," J Exp Psychol Hum Percept Perform, 9(6). 912-922. 1983.

[25] Abrams, R.A., Jonides, J, "Programming saccadic eye movements,” J Exp Psychol Hum Percept Perform, 14(3). 428-443. 1988.

[26] Inhoff, A.W, "Preparing sequences of saccades under choice reaction conditions: effects of sequence length and context," Acta Physiol, 61(3). 211-228. 1986.

[27] Zingale, C.M., Kowler, E, "Planning sequences of saccades," Vis Res, 27(8). 1327-1341. 1987.

[28] Crawford, T.J, "Multi-stepping saccadic sequences in humans," Acta Physiol, 76(1). 11-29. 1991.

[29] Jacobs, A.M, “On localization and saccades programming," Vis Res, 27(11). 1953-1966. 1987.

[30] Nazir, T.A., Jacobs, A.M, "The effects of target discriminability and retinal eccentricity on saccade latencies: an analysis in term of variable-criterion theory,” Psychol Res, 53(4). 281-289. 1991.

[31] Biscaldi, M., Fischer, B., Stuhr, V, "Human express-saccade makers are impaired at suppressing visually-evoked saccades,” J Neurophysiol, 76(1). 199-214. 1996.

[32] Fischer, B., Boch, R, "Saccadic eye movements after extremely short reaction times in the monkey," Brain Res, 260(1). 21-26. 1983

[33] Cavegn, D., Biscaldi, M, "Fixation and saccade control in expresssaccade maker,” Exp Brain Res, 109(1). 101-116. 1996.

[34] Sakata, H., Shibutani, H., Kawano, K, "Spatial properties of visual fixation neurons in posterior parietal association cortex of the monkey,” J Neurophysiol, 43(6). 1654-1672. 1980.

[35] Goldberg, M.E., Bushnell, M.C., Bruce, C.J, "The effect of attentive fixation on eye movements evoked by electrical stimulation of the frontal eye fields,” Exp Brain Res, 61(3). 579584. 1986.

[36] Biscaldi, M., Gezeck, S., Stuhr, V, "Poor saccadic control correlates with dyslexia," Neuropsychol, 36(11). 1189-1202. 1998.

[37] McConkie, G.W., Kerr, P.W., Reddix, M.D., Zola, D, "Eye movement control during reading: I. The location of initial eye fixation in words," Vis Res, 28(10). 1107-1118. 1988. 
[38] O’Regan, J.K, “The control of saccade size and fixation duration in reading: The limits of linguistic control," Percept Psychophys, 28(2). 112-117. 1980.

[39] McConkie, G.W, Eye movements and perception during reading, in: Eye movements in reading. Perceptual and Language processes, Rayner Editor, Academic Press Publishers, New York, 1983.

[40] Rayner, K., Pollatsek, A, The psychology of reading, Englewood Cliffs Editor, Prentice Hall Publishers, Upper Saddle River, 1989.

[41] Adler-Grinberg, D., Stark, L, "Eye movements, scan paths, and dyslexia,” American J Optom Physiol Opt, 55(8). 557-570. 1978.

[42] Brown, B., Haegerstrom-Portnoy, G., Adams, A.J., Yingling, C.D., Galin, D., Herron, J., Marcus, M, "Predictive eye movements do not discriminate between dyslexic and control children," Neuropsychologia, 21(2). 121-128. 1983.

[43] Brown, B., Haegerstrom-Portnoy, G., Yingling, C.D., Herron, J., Galin, D., Marcus, M, "Tracking eye movements are normal in dyslexic children,” Am J Optom Phys Opt, 60(5). 376-383. 1983.

[44] Stanley, G., Smith. G.A., Howell, E.A, "Eye-movements and sequential tracking in dyslexic and control children,” Br J Psychol, 74(Pt2). 181-187. 1983.

[45] [Stanley, G., Smith, G.A., Howell, E.A, "Eye-movements in dyslexic children: comments on Pavlidis reply,” Br J Psychol, 74(Pt2). 195-197. 1983.
[46] Black, J.L., Collins, D.W., De Roach, J.N., Zubrick, S, “Dyslexia: saccadic eye movements,” Percept Mot Skills, 58(3). 903-910. 1984.

[47] Black, J.L., Collins, D.W., de Roach, J.N., Zubrick, S, “A detailed study of sequential saccadic eye movements for normal- and poorreading children,” Percept Mot Skills, 59(2). 423-434. 1984.

[48] Rayner, K, "Do faulty eye movements cause dyslexia?," Developmental Neuropsychology, 1(1). 3-15. 1985.

[49] Olson, R.K., Kliegl, R., Davidson, B.J, "Dyslexic and normal readers'eye movements,” J Exp Psychol Hum Percept Perform, 9(5). 816-825. 1983.

[50] Olson, R.K., Conners, F.A., Rack, J.P, Eye movements in dyslexic and normal readers, in: Vision and Visual Dyslexia, Stein Editor, Macmillian Press Publishers, London., 1991.

[51] Aleci, C., Piana, G., Piccoli, M., Bertolini, M, "Developmental dyslexia and spatial relationship perception,” Cortex, 48(4). 466476. 2012.

[52] Aleci, C., Piana, G., Anselmino, F, "Evaluation of Spatial Anisotropy by Curvature Analysis of Elliptical Targets,” Open Ophthalmol J, 4. 20-26. 2010.

[53] Rubin, G.S., Feely, M, "The role of eye movements during reading in patients with age-related macular degeneration (AMD),” Neuro-Ophthalmology, 33(3). 120-126. 2009. 